\title{
Bioimpresoras 3D como herramienta de innovación en el futuro de trasplantes de órganos
}

\author{
$3 D$ bioprinters as innovation tool in the future of organ transplants \\ Bioimpresoras 3D como uma ferramenta para a inovação no futuro de \\ transplantes de órgãos
}

Carmen Gómez Aranda

Centro Universitario Temascaltepec, Universidad Autónoma del Estado de México, México

dianakelsi@hotmai.com

\section{Resumen}

El presente trabajo tuvo por objetivo demostrar que la Bioimpresora 3D tendrá un impacto positivo como herramienta de innovación en el futuro de trasplante de órganos en el año 2016. La idea se basa en la investigación documental ya que es una técnica que consiste en analizar, seleccionar y recopilar información, mediante la lectura de documentos y materiales bibliográficos que contienen datos relacionados con el estudio.

Esta nueva tecnología tendrá un impacto positivo en la sociedad. Diversos estudios de diferentes autores han concluido que es una herramienta muy útil para solucionar la falta de cultura en la donación de órganos, la larga espera para ser candidato a un trasplante por donación y el riesgo de que sea rechazado por nuestro cuerpo.

Palabras clave: medicina, impresión 3D, trasplante de órganos. 


\section{Abstract}

This work was aimed at demonstrating that the $3 \mathrm{D}$ Bioprinter will have a positive impact as a tool for innovation in the future of organ transplantation in 2016. The idea is based on documentary research since it is a technique that is to analyze, select and collect information through the reading of documents and bibliographic materials containing data related to the study.

This new technology will have a positive impact on society. Several studies from different authors have concluded that it is a very useful tool to solve the lack of culture in organ donation, the long wait to be candidate for a transplant by donation and the risk of that is rejected by our body.

Key Words: medicine, organ transplantation, 3D printing.

\section{Resumo}

Este estudo teve como objetivo demonstrar que o bioprinter 3D terá um impacto positivo como uma ferramenta para a inovação no futuro do transplante de órgãos em 2016. A ideia é baseado em pesquisa documental, pois é uma técnica que envolve análise, selecione e recolher informação através da leitura de documentos e materiais de biblioteca que contêm dados relacionados com o estudo.

Esta nova tecnologia terá um impacto positivo na sociedade. Vários estudos realizados por diferentes autores concluíram que é uma ferramenta muito útil para resolver a falta de cultura em doação de órgãos, a longa espera para ser um candidato para uma doação de transplantes e o risco de ser rejeitado pelo organismo.

Palavras-chave: medicina, impressão em 3D, transplante de órgãos.

Fecha recepción: Enero 2016

Fecha aceptación: Junio 2016 


\section{Introducción}

La Oficina Europea de Patentes (OEP) anunció que el ganador del premio al Inventor Europeo 2014, considerado el Óscar de los inventores, era Charles W. Hull de Estados Unidos, el hombre que creó la primera impresora 3D, una tecnología con un gran potencial futurista (Saber Mas, 2014).

Esta es una de las tecnologías de creciente incorporación e impacto que se utiliza actualmente para indicar la aparición de nuevas alternativas en la medicina con el fin de mejorar la calidad de vida de las personas: nanotecnología, biotecnología, tecnologías de información y comunicación, robótica e inteligencia artificial. Esta unión ha permitido crear Impresoras de órganos en 3D, lo que dado lugar a diversas prácticas en cirugía de cerebro, elaboración de prótesis y remplazo de órganos. Las Impresoras 3D están siendo empleadas en el área de la medicina para mejorar y preservar la calidad del paciente.

En el mundo, más de mil millones de personas tienen alguna deficiencia y cerca de 200 millones presentan algún tipo de problema funcional, cifras que están aumentando preocupantemente. Por tanto, requieren de apoyo para mejorar su calidad de vida.

Sin duda, las Bioimpresoras 3D han llegado para quedarse, generando opiniones diversas. Cada vez se habla más de ellas y su rápida evolución hará que un día no muy lejano sea común tener una en casa, como si fuese una impresora de papel. Pero, ¿cuál es el impacto que provocará su uso? En este artículo se describen las diferentes opiniones de los investigadores del área. Las tecnologías de Impresión 3D se basan en las denominadas "tecnologías de procesos aditivos", un concepto de fabricación aditiva que describe las tecnologías en las que un objeto es creado mediante la definición de una secuencia en capas. La Bio-printing 3D se está utilizando en la medicina regenerativa para combatir la necesidad de tejidos y órganos adecuados en trasplantes. En comparación con la impresión no biológica, Bio-printing 3D implica complejidades adicionales, tales como la elección de materiales, tipos de células, factores de crecimiento y diferenciación, y desafíos técnicos relacionados con la sensibilidad de las células vivas y la construcción de tejidos (Chimbo, 2016). 


\section{Antecedentes.}

Aunque es sorprendente lo que nos ofrece esta tecnología en tercera dimensión, este invento no es tan nuevo ya que el primero se produjo el 9 de marzo de 1983, al imprimir una copa de plástico negro. Hulls creó la empresa 3D Systems en 1986, tres años después de su primera impresión, en Valencia (California). Esta tecnología fue creada para hacer objetos de plástico y poner a prueba prototipos (Saber Mas, 2014).

La técnica utilizada fue la estereolitografía, que designa un sistema de fabricación capa por capa con aditivo sensible a los rayos UV conocido como impresora 3D laser. En 2009, este movimiento comenzó en todo el mundo con el inicio de la empresa www.sculpteo.com que desarrolla herramientas web que simplifican todo el proceso de la impresora 3D para aficionados. Al mismo tiempo, Makerbot Industria fundada por Bree Pettis, lanzó Makerbot Cupcake CNL para particulares, así que otros actores se involucraron en el campo de las impresoras 3D historia. El proyecto RepRap (replicador Rapid Prototyping) es un proyecto británico de la Universidad de Bath para crear una impresora 3D en parte auto-replicativa (es decir, sin patente y con planos disponibles gratuitamente para todo el mundo en internet). El primer modelo operativo, Darwin, lanzado en 2007, era totalmente código abierto, lo que permitía a los usuarios apasionados el ensamblarlo y mejorarlo en casa.

El real despegue de la impresión en 3D ocurrió en 2011, cuando 15000 impresoras 3D se vendieron entre los 40 modelos que ya están disponibles en el mercado. A partir de 2014, la impresión en 3D evolucionó de la manufactura instantánea de objetos domésticos a generar tejidos orgánicos a partir de bases celulares. La realidad es que ya podemos hacer cosas que parecen de ciencia ficción. Estamos en el antesala de la tercera revolución industrial de la historia (Mora, 2015).

La impresión en tercera dimensión (3D) provocará en los siguientes años una nueva revolución industrial, por lo que HP Inc. se enfocará en este mercado a partir de 2016, y México será parte de los países que tengan primero esta tecnología (Hernández, 2015).

Objetivo general: demostrar que la Bioimpresora 3D tendrá un impacto positivo como herramienta de innovación en el futuro de trasplantes de órganos. 


\section{Metodología documental}

La investigación documental es una técnica que consiste en la selección y recopilación de información por medio de la lectura y crítica de documentos y materiales bibliográficos. Franklin (1997) define la investigación documental como una técnica de investigación en la que se deben seleccionar y analizar aquellos escritos que contienen datos de interés relacionados con el estudio.

El desarrollo de un proceso de investigación documental completo da como producto diferentes tipos de trabajos documentales entre los que se encuentran compilaciones, ensayos, críticas valorativas, estudios comparativos, memorias, monografías, entre otros (Baray, 2015).

Al llevar a cabo este proceso de investigación se elaboró un proyecto que comunica con claridad y coherencia los resultados, descubrimientos, comprobaciones o reflexiones logrados a lo largo de todo el proceso de investigación documental (Rivera, 2008).

\section{Materiales y métodos}

La información necesaria para el desarrollo de este artículo se toma de la recolección de tesis, investigaciones procedentes de instituciones tecnológicas y artículos científicos que tratan el tema. La metodología a utilizar es documental ya que la información se selecciona de diferentes sitios por medio de la lectura de documentos, materiales. Más adelante mediante un proceso se presenta un punto de discusión (Martínez, 2002).

\section{Definición de innovación}

Se establece que la innovación es la introducción de un nuevo, o significativamente mejorado producto (bien o servicio), de un proceso, método de comercialización o de un nuevo método organizativo al mercado y la sociedad (Schumpeter, 2005).

La innovación es definida también como el proceso que permite conjugar habilidades y técnicas en función de dar soluciones novedosas a problemas particulares (Fagerber, 2005). 


\section{¿Qué es el trasplante de órganos?}

Es un tratamiento médico indicado cuando toda otra alternativa para recuperar la salud del paciente se ha agotado, que sólo es posible gracias a la voluntad de las personas que dan su consentimiento para la donación. Se trata del reemplazo de un órgano vital enfermo, sin posibilidad de recuperación, por otro sano. Los órganos que se trasplantan en nuestro país son: riñón, hígado, corazón, pulmón, páncreas e intestino. La donación de órganos es posible sólo en 5 de cada 1000 fallecimientos debido a la complejidad de los requerimientos necesarios para llevarla a cabo; sólo puede concretarse si la muerte sucede en una unidad de terapia intensiva y requiere de inmediato una serie de pasos coordinados en simultáneo (Proyecto Salud, 2013).

\section{Definición de Impresora 3D}

Una impresora 3D es un dispositivo capaz de generar un objeto sólido tridimensional mediante (y ahí radica la principal diferencia con los sistemas de producción tradicionales) la adición de material. Los métodos de producción tradicionales son sustractivos, es decir, generan formas a partir de la eliminación de exceso de material. Las impresoras se basan en modelos 3D para definir qué se va a imprimir. Un modelo no es sino la representación digital de lo que vamos a imprimir mediante algún software de modelado (Chimbo, 2016).

\section{Definición de Bioimpresora 3D}

La impresión en 3D permite, desde hace algunos años, fabricar objetos apilando delicadamente capas de resina o de plástico. Unos biólogos tuvieron la gran idea de reemplazar el plástico por células para lograr crear estructuras orgánicas; la función es la misma Impresión en 3D, sólo que imprime tejidos y órganos humanos.

El proceso de impresión utiliza un polímero médico, materiales inorgánicos y gel de agua mezclado con células vivas, todo se conjunta en condiciones asépticas con lo que obtienen un tejido con $90 \%$ de células vivas que han sido capaces de mantenerse con vida durante máximo cuatro meses (Gaona, 2014). 


\section{Imprimir en 3D.}

Esta es una tecnología propia de arquitectos e ingenieros que cumplieron su sueño de materializar sus diseños directamente desde la computadora, con impresoras que colocan capa por capa el material deseado en el lugar indicado. Ahora bien, de maquetas a órganos hay un gran trecho, pero no lo suficientemente largo como para evitar que Mironov y el doctor Gabor Forgacs, de la Universidad de Missouri en Estados Unidos, adaptaran esta tecnología a la medicina regenerativa y de trasplantes (Carnevale, 2010).

La bio-impresora 3D se usará en hospitales, laboratorios, institutos y universidades, y será tan esencial como es hoy en día un multímetro para un electrónico o un horno para un panadero (García, 2015).

Esto se debe a las posibilidades que ofrecen estas máquinas en cuanto a biofabricación. Algunas de las aplicaciones inmediatas se describen a continuación.

$\checkmark$ Fabricación de arreglos de gotas para cultivos.

$\checkmark$ Fabricación de andamios celulares con morfología y porosidad variable.

$\checkmark$ Fabricación de estructuras tridimensionales con múltiples fluidos.

$\checkmark$ Impresión directa de células vivas embebidas en fluidos biocompatibles (García, 2015).

\section{¿En qué consiste la de Bioimpresión 3D?}

El bio-printing o bio-impresión es una técnica que se centra en la identificación de estructuras y elementos que componen tejidos específicos $y$, con base en ello, en la creación de un diseño que pueda generar tejidos de laboratorio a través de bioimpresoras especializadas. Una vez que se tiene el diseño, el siguiente paso es desarrollar los protocolos de bioprocesos requeridos para generar varios bloques de células, también conocidos como biotintas, que se utilizarán para construir el tejido, suplantando a los polímeros plásticos de las impresoras 3D convencionales. Los bloques de biotintas son creados a partir de una bioimpresora, que capa a capa desarrollará el tejido final a manera de vivero donde crecerá el material celular. Componentes como el hidrogel es utilizado como soporte de los tejidos para construir verticalmente la tridimensionalidad, o bien sirven como material de relleno para canales o espacios vacíos dentro de los tejidos. Los procesos de bio-printing se pueden adaptar a una gran variedad de formatos, desde tejidos a micro-escala hasta el cultivo de estructuras más grandes (Gaona, 2014). 


\section{¿Cómo funciona una Bioimpresora 3D?}

La impresión de órganos humanos tiene varias etapas. Primero, los científicos toman muestras de tejidos o células madre del paciente, las que se cultivan en laboratorio esperando que se multipliquen. A continuación, estas células se transforman en una especie de tinta biológica, que es la que se utiliza en la bioimpresión. Las impresoras están programadas para crear diferentes órganos, todo a la medida de lo que requiere el paciente. Se imprime un modelo de estas células, ya diferenciadas para su uso, en tercera dimensión, para luego implantarlas en el cuerpo humano, esperando que se fundan con las células ya existentes, reemplazando al órgano que falla.

No se imprime un órgano tal como lo conocemos, sino son conjuntos celulares capaces de realizar los procesos que no están funcionando por el fallo del órgano. Incluye dos cabezas de impresora, una para colocar células humanas y la otra para colocar un hidrogel, trama o matriz de soporte. La cabeza de impresión celular forma las células en gotas de $100 \mu \mathrm{m}$ - $500 \mu \mathrm{m}$ de diámetro, cada una con 10 000-30 000 células (Valenzuela, 2014).

Las gotas retienen su forma bien y pasan fácilmente a través del proceso de impresión de inyección de tinta. La segunda cabeza de inyección se usa para depositar un hidrogel a base de azúcar usado como andamio, que no interfiere con las células o se adhiere a ellas. Una vez que la impresión se ha completado, la estructura es dejada durante un día o dos para permitir que las gotas se fusionen. Para estructuras tubulares como los vasos sanguíneos, el hidrogel se imprime en el centro y en el exterior del anillo de cada sección transversal antes de que las células sean añadidas.

Un sistema de calibración basado en láser, controlado por computador, es usado para posicionar repetidamente la punta capilar unida a la cabeza de la impresora, asegurando que las células sean colocadas en exactamente la posición correcta dentro de un micrómetro de tolerancia. La bioimpresora 3D incluye una interfaz de software que permite a los ingenieros construir un modelo

de la construcción de un tejido antes de que la impresora comience las construcciones físicas de los órganos, célula por célula (Valenzuela, 2014).

\section{Los retos de la Bioimpresión 3D}

En teoría suena bien, pero los retos no son fáciles. José Becerra, catedrático de Biología Celular en la Universidad de Málaga y director científico del Centro Andaluz de Nanomedicina y Biotecnología ( BIONAND), hace eco de las dificultades a solucionar. 
Por el lado biológico existe la tecnología y el conocimiento, basado en las células madre y la ciencia de los materiales. Es decir, existen los materiales, las células y la posibilidad de cultivarlas, solamente resta conjuntarlas.

$\checkmark$ Para hacer esto hay que calcular de forma precisa cómo se lleva a cabo el proceso, cuál es el momento adecuado para ponerlo en marcha, qué cantidad de células hay que inyectar o cuánto tiempo se tienen en cultivo en el laboratorio antes de ser implantadas. También es imprescindible tener muy claro cómo se alimentan las células en el tiempo en que la pieza esté cultivándose en el laboratorio. Esta es una cuestión básica.

$\checkmark$ Las células tienen que vivir cada minuto y por tanto cada minuto les tienen que llegar nutrientes. La llegada de los nutrientes en un sistema 3D es compleja porque tienen que atravesar por difusión esa estructura 3D. Esto sólo es posible con unas máquinas llamadas biorreactores, que son capaces de incluir los nutrientes en un líquido. Los biorreactores introducen oxígeno, y retiran el dióxido carbónico y los otros restos del metabolismo de la célula. Esto es necesario desde que se pone en marcha el cultivo hasta que se implanta en el cuerpo humano.

$\checkmark$ Esas células que hasta ahora se han alimentado en el bio-reactor ahora tienen que alimentarse en el organismo receptor. Esto quiere decir que la sangre del organismo tiene que llegar a todas esas células que han vivido hasta ahora en un ambiente especialmente diseñado para que sigan vivas, explica el director científico del centro BIONAND.

$\checkmark$ Conseguir la vascularización, la creación de vasos sanguíneos que lleven los nutrientes a las células implantadas es el verdadero reto. Se pueden cultivar células en diferentes sustratos en el laboratorio, así como promover la creación de pequeños vasos sanguíneos (Bejerano, 2014).

\section{Ventajas y desventajas de las Bioimpresoras 3D}

\section{Ventajas}

$\checkmark$ Versatilidad. La revolución que se supone para la manufactura de productos. Una sola impresora 3D es capaz de realizar una infinidad de productos distintos. Gran parte de la manufactura actual se realiza con máquinas específicas cuya función es limitada y si el producto cambia la máquina también debe re adaptarse o cambiarse. 
$\checkmark$ Flexibilidad y prototipado rápido. El límite es la imaginación y la capacidad para representar tus ideas en 3D. Permite realizar prototipos de productos con facilidad, lo que puede implicar una mejora en el diseño de estos.

$\checkmark$ Reducción de costes. Tanto en el proceso de producción como en el proceso de transporte. La producción puede realizarse desde casa.

$\checkmark$ Personalización. Una de las ventajas más atractivas es la posibilidad de realizar tus propias prendas, productos, objetos de forma personalizada y exclusiva.

$\checkmark$ Nuevas industria y sector. Nueva industria y nuevo sector que crearán nuevas formas de negocio.

$\checkmark$ Aplicaciones múltiples aún por descubrir. En el campo de la medicina encontramos aplicaciones sorprendentes, por ejemplo, la creación de prótesis o incluso la impresión de tejidos orgánicos Artículo sobre impresión de tejidos. La impresión 3D tiene mucho campo por recorrer y cada vez se aplicará en más campos.

\section{Desventajas}

$\checkmark$ Vulnera los derechos de autor. La réplica de objetos con copyright será difícil de controlar pues los escáneres 3D permiten la réplica de cualquier objeto.

$\checkmark$ Usos malintencionados. Lamentablemente existe la posibilidad de crear objetos tales como armas de fuego y el peligro de generalizar este tipo de objetos.

$\checkmark$ Aumento de productos inútiles. Hay que reconocerlo, yo mismo puedo ponerle empeño para realizar algún modelo 3D, puedo hacerlo más bonito o más feo, pero la pregunta que uno se tiene que hacer antes de ponerse a imprimir nada es: ¿me va a servir para algo? (Mejía, 2016).

\section{Preocupaciones éticas sobre la Bioimpresión 3D}

$\checkmark$ Sin embargo, no todos están cómodos con la nueva habilidad de crear partes humanas en un laboratorio.

$\checkmark$ Un director de investigación en Gartner Inc., la empresa de investigación y consultoría en tecnología de información, cree que la bioimpresión 3D avanza tan rápidamente que incitará un debate ético de gran escala en el 2016. 
$\checkmark$ Instalaciones dedicadas a la bioimpresión 3D de órganos y tejidos humanos avanzarán mucho más rápido que la comprensión y aceptación general de las ramificaciones de esta tecnología, dijo Pete Basiliere en un reporte reciente.

$\checkmark$ Estas iniciativas tienen buenas intenciones, pero generan un número de preguntas que siguen sin respuesta, agregó Basiliere. ¿Quién controlará la habilidad para producirlos? ¿Quién asegurará la calidad de estos productos?

$\checkmark$ Es probable que estos órganos bioimpresos sean costosos, lo cual los pondría fuera del alcance de todos, menos los pacientes más ricos.

$\checkmark$ Murphy comentó que Organovo sólo usa células humanas para crear tejidos, y no ve ningún problema ético con las actividades de su compañía.

$\checkmark$ La gente solía preocuparse por hacer investigación con cadáveres y eso terminó rápidamente.

$\checkmark$ Creen que el siguiente paso será la impresión de tiras de tejido, o parches, que se podrían utilizar para reparar hígados y otros órganos dañados.

$\checkmark$ Claro, cualquier uso de tejidos bioimpresos en procedimientos quirúrgicos necesitaría la aprobación de la FDA (Administración de Alimentos y Medicamentos de Estados Unidos, por sus siglas en inglés). Para ese entonces, la noción de un cirujano utilizando un riñón bioimpreso en un paciente ya no sería algo tan extraño. Sin embargo, esta tecnología podría crear nuevas interrogantes morales.

Las preguntas éticas están destinadas a ser las mismas preocupaciones que hemos visto en el pasado. Muchos avances médicos han sufrido resistencias morales, desde trasplantes de órganos hasta la investigación con células madre. ¿Sólo los ricos serán capaces de acceder a esto? ¿Estamos jugando a ser Dios? Al final, salvar vidas tiende a triunfar sobre cualquier objeción (Griggs, 2014).

El previsible impacto de la impresión 3D en la medicina es uno de los principales factores que invita a pensar que será posible crear órganos y tejidos a medida, compatibles con el receptor a partir de células obtenidas del propio paciente. Una prueba de ello son los equipos capaces de fabricar tejido hepático vivo, que ya son una realidad, como muestra el catálogo de la empresa estadounidense Organovo, una de las líderes del sector (Prats, 2013). 
Métodos y técnicas que dan resultados favorables a esta innovadora tecnología, llamada Bioimpresora 3D

Se demuestran los contados trasplantes de este tipo que se han realizado hasta la fecha, cuando ya no hay más alternativa y se utiliza la tecnología como ultima opción.

Fue muy sonado el caso de Hannah Warren, una niña de dos años que sufría una rara enfermedad (agnesia traqueal congénita) que le impedía alimentarse por la boca, hablar o respirar con normalidad, porque su tráquea no se había desarrollado. Fue la persona más joven del mundo en recibir un trasplante de tráquea bioartificial, a partir de fibras de plástico a las que se añadieron las células propias de la niña, extraídas de su médula ósea. Esto sucedió el 9 de abril de 2013 (el responsable de la intervención: Paolo Macchiarini (Tardón, 2016).

En palabras de Julio Acero, jefe del Servicio de Cirugía Oral y Maxilofacial del Hospital Universitario Ramón y Cajal de Madrid, el avance de Atala y sus compañeros consiste en una combinación de impresión en tres dimensiones con ingeniería tisular. En la actualidad, la impresión tridimensional la empleamos de forma rutinaria en nuestro taller de anaplastología, para hacer implantes craneales, reconstrucción de guías para reparar la mandíbula, placas para fijar los colgajos. El objetivo de unir el crecimiento celular con la impresión 3D, agrega este especialista, es dar una estructura más viva al órgano, en este caso la oreja, con su piel, su cartílago (Tardón, 2016).

El propio Atala subraya que la nueva tecnología permitiría elaborar tejidos vivos y órganos para implantarlos quirúrgicamente. La oreja no es el único órgano artificial con el que se está trabajando en laboratorio, se están creando otros prototipos de riñones, vejigas, piel, huesos, corazón. Aunque estamos en una fase inicial, argumenta Becerra, la bioimpresión es una vía muy prometedora. Sin duda, la medicina regenerativa para la creación de órganos es una opción muy esperanzadora, con la que en un futuro podrían beneficiarse los pacientes que están en lista de espera de un trasplante (Tardón, 2016).

Este prometedor método no es el único en el que se está trabajando en laboratorio para reducir las listas de espera de trasplantes y evitar el rechazo de éstos. Entre otras utilidades, existen otras dos fórmulas que también podrían tener éxito en el futuro, la descelularización de órganos y el uso de órganos animales, de la primera técnica existe una experiencia en Estocolmo, que consiste en 
el lavado de órganos para eliminar las células del donante y evitar así una respuesta inmunológica del paciente receptor al recibir el implante. En cuanto al uso de órganos animales, el nombre del español Juan Carlos Izpisúa es clave, este científico dirige una investigación que pretende desarrollar órganos humanos en el interior de cerdos. El proyecto se está llevando a cabo en una granja de Murcia (Tardón, 2016).

Un grupo de cirujanos de un hospital de Nueva York ha destacado la relevancia que tuvo la capacidad de imprimir un corazón en 3D para salvar la vida de un pequeño bebé de dos semanas que necesitaba ser sometido a una complicada operación quirúrgica. Este grupo de doctores imprimió un corazón 3D a partir de los datos obtenidos en una imagen de resonancia magnética del corazón del bebé. Dicho órgano estaba muy dañado y esa reproducción del órgano en 3D permitió a los doctores estudiarlo y desarrollar una estrategia detallada antes de operar. Esto supone un avance notable para las cardiopatías congénitas y vuelve a demostrar la capacidad que tiene la impresión 3D de ser un elemento importante en todo tipo de tratamientos y procedimientos médicos (Pastor, 2014).

\section{Aportación del conocimiento y comprensión adquirido durante la investigación sobre la}

\section{Bioimpresora 3D en el futuro de trasplante de órganos}

Como bien pudimos observar en las investigaciones anteriores sobre los métodos y técnicas de Impresión 3D en el campo de la medicina, todas las publicaciones que existen sobre trasplantes son de impacto positivo, dieron resultados favorables a las personas que se sometieron a ellos como último recurso y sabiendo que fueron creados mediante una Bioimpreora 3D.

Los casos reales aún son pocos, pero se está trabajando en ello para mejorarlos y mostrar a la sociedad que pueden suplir de una forma eficaz y segura un órgano donado por otra persona, con la ventaja de que éste no tiene la probabilidad de ser rechazado por el cuerpo receptor pues fue creado con sus propias células, dando un porcentaje casi nulo de rechazo por parte del cuerpo. Los especialistas encargados de esta nueva tecnología hacen ver que está avanzando a pasos agigantados día con día, y que viene a cambiar el método de obtención de algún órgano o parte del cuerpo para ser trasplantado y dejar atrás el difícil proceso de enlistarse y ser aceptado como candidato para algún tipo de donación de órganos. 


\section{Conclusiones}

La impresión 3D está presente en casi todas las áreas y ha demostrado que tiene un incalculable futuro. Nos hace visualizar el presente y preguntarnos sobre la gran importancia que tiene esta innovadora herramienta tecnológica en nuestras vidas.

La conclusión es que la tecnología de Bioimpresoras 3D tendrá un impacto positivo en la sociedad, ya que gracias a su uso se podrán salvar millones de vidas con la creación de órganos; algunas partes del cuerpo pueden ser remplazadas con células vivas del paciente.

Aún hay un largo camino que recorrer ya que existen obstáculos en las tecnologías celular, de bioproducción e integración, las cuales juegan un papel importante para la separación de células madre en trasplantes. Los avances tecnológicos son prueba de que todo se puede y la imaginación es el único límite.

\section{Bibliografía}

Baray, H. L. (14 de diciembre de 2015). Introducción a la Metodología de la Investigación. Obtenido de Introducción a la Metodología de la Investigación: http://www.eumed.net/libros-gratis/2006c/203/\#indice

Bejerano, P. G. (09 de octubre de 2014). Bioimpresión 3D lo que hay y lo que vendrá. Obtenido de Bioimpresión 3D lo que hay y lo que vendrá: http://www.eldiario.es/turing/bioimpresion-3d_0_311119086.html

Carnevale, R. (2010). La impresora de órganos. Funciones y realidades de la producción de tejidos artificiales. Química Viva, 10.

Chimbo, K. M. (2016). Los Beneficios de las Impresoras 3D como Herramienta de Innovación en la Medicina. Caribeña de Ciencias Sociales, 11.

Fagerber. (2005). Innovación y Tecnología. Obtenido de Innovación y Tecnología: http://www.lasalle.edu.co/wps/wcm/connect/b98917e3-b2a2-4a70-ae99cfdc56850ddc/innovaci\%C3\%B3n+y+Tecnolog\%C3\%ADa.pdf?MOD=AJPERES

Gaona, A. G. (14 de marzo de 2014). Siguiente paso de la Impresión 3D. El Cuerpo Humano. Obtenido de Siguiente paso de la Impresión 3D. El Cuerpo Humano: 
http://www.mediatelecom.com.mx/index.php/tecnologia/item/61005-siguiente-paso-dela-impresion-3d-el-cuerpo-humano

García, D. A. (2015). Diseño y construcción de una Bioimpresora 3D. En D. A. García, Diseño y construcción de una Bioimpresora 3D. (pág. 92).

Griggs, B. (03 de abril de 2014). El próximo paso de la Impresión 3D. Obtenido de El próximo paso de la Impresión 3D: http://expansion.mx/tecnologia/2014/04/03/el-proximo-paso-enla-impresion-en-3d-los-organos-humanos

Hernández, A. (17 de noviembre de 2015). EXCELSIOR. La Impresión en 3D, negocio en evolución., pág. http://www.excelsior.com.mx/hacker/2015/11/27/1059912. Obtenido de EXCELSIOR

Martínez, S. (12 de agosto de 2002). Grupo Emergente de Investigación Oaxaca. Obtenido de Grupo Emergente de Investigación Oaxaca: http://geiumaoax.net/invdoc/importanciaydef.htm

Mejía, N. (31 de agosto de 2016). Impresoras 3D en Medicina. Obtenido de Impresoras 3D en Medicina: http://impresoras3denmedicina.blogspot.mx/2016/08/ventajas-y-desventajasde-la-impresion.html

Mora, A. (14 de mayo de 2015). 3D Market. Obtenido de 3D Market: https://www.3dmarket.mx/articulos/impresoras-3d-historia/

Pastor, J. (7 de octubre de 2014). Xataka. Obtenido de Xataka: http://www.xataka.com/makers/un-corazon-impreso-en-3d-ayuda-a-salvar-la-vida-de-unbebe-de-dos-semanas

Prats, J. (12 de noviembre de 2013). El País. Obtenido de El País: http://sociedad.elpais.com/sociedad/2013/11/12/actualidad/1384289478_726164.html

Proyecto Salud. (17 de octubre de 2013). Obtenido de Proyecto Salud: http://www.proyectosalud.com.ar/shop/detallenot.asp?notid=7810

Rivera, R. F. (16 de julio de 2008). mail.com. Obtenido de mail.com: http://www.mailxmail.com/curso-investigacion-documental/proceso-investigaciondocumental

Saber Mas. (17 de agosto de 2014). Obtenido de Saber Más: http://www.sabermas.umich.mx/archivo/secciones-anteriores/tecnologia/125-numero16/252-la-impresion-en-3d.html 
Schumpeter. (2005). Innovación y Tecnología. Obtenido de Innovación y Tecnología: http://www.lasalle.edu.co/wps/wcm/connect/b98917e3-b2a2-4a70-ae99cfdc56850ddc/innovaci\%C3\%B3n+y+Tecnolog\%C3\%ADa.pdf?MOD=AJPERES

Tardón, L. (15 de febrero de 2016). El Mundo. Obtenido de El Mundo: http://www.elmundo.es/salud/2016/02/15/56c1f71322601d12128b4582.html

Valenzuela, I. (28 de mayo de 2014). Futuro de la Impresión 3D: Órganos humanos. Obtenido de Futuro de la Impresión 3D: Órganos humanos: http://www.vix.com/es/btg/curiosidades/5985/el-futuro-de-la-impresion-3d-organoshumanos 